\title{
IRB review points for studies utilizing paraffin blocks archived in the pathology laboratory
}

\author{
Yong-Jin Kim ${ }^{1}$, Chang Rok Jeong ${ }^{2}$, Jeong Sik Park ${ }^{3}$ \\ ${ }^{I}$ Department of Pathology, Kyungpook National University School of Medicine; ${ }^{2}$ Department of Ethics Education, Kyungpook National \\ University Teachers College; ${ }^{3}$ Department of Philosophy, Kyungpook National University College of Humanities, Daegu, Korea
}

In the personalized medicine era, utilizing paraffin blocks in pathology archives for investigating human diseases has come into the limelight. This archived material with clinical data will reduce the research time and could prevent new patient recruitment to obtain tissue for research. However, the clause indicating the necessity of consent from human material providers in the Korean Bioethics and Safety Act has made the Institutional Review Board (IRB) deny permission to use paraffin blocks for research without consent, and alternatively to get the same before starting an experiment. Written consent may be waived off in studies using paraffin blocks with anonymous status or conditions not linked to personal information by applying the paragraph 3, article 16 of the current Bioethics and Safety Act. Also, the IRB should recommend researchers to preserve the blocks as medical records of patients in long-term archives.

Keywords: Paraffin block; The Bioethics and Safety Act; IRB; Human biological material; Consent

\section{INTRODUCTION}

Paraffin blocks archived in the department of pathology are made from patient tissues obtained for diagnostic or therapeutic purposes. Excised tissues from different clinical departments are transferred to the pathology department, where the specimen is examined by a pathologist and sections with diagnostic significance are selected and obtained. These sections eventually turn into paraffin blocks after going through several histologic processes, including dehydration and embedding. Slides for microscopic examination are made when 4-6- $\mu \mathrm{m}$ thickness ribbons from these paraffin blocks are at-

Received: April 5, 2018, Revised: June 10, 2018

Accepted: June 11, 2018

Corresponding Author: Yong-Jin Kim, Department of Pathology, Kyungpook National University School of Medicine, 130, Dongdeok-ro, Jung-gu, Daegu 41944, Korea

Tel: +82-53-200-5250, Fax: +82-53-426-1525

E-mail: yjjjkim1@gmail.com tached onto a glass slide and stained. Paraffin blocks are reusable for different examinations (i.e. special staining or reexamination) and therefore managed and stored in the department of pathology. In addition, these blocks can serve as legal evidences supporting the fact that the patient has undergone surgical treatment. Hence, these blocks are considered as medical records and are usually stored for at least 5 years in majority of the hospitals.

Recent advancements in research methods have allowed diverse studies using paraffin blocks, including the extraction of deoxyribonucleic acid (DNA) from these blocks to perform genetic testing for oncogene identification and therapeutics. Furthermore, a key advantage is that prospective tissue collection can be avoided by utilizing long archived material, dramatically shortening the study period. More specifically, follow-up medical records can also be used in parallel, and therefore paraffin blocks have become an important study material for personalized medicine.

Since paraffin blocks are human derivatives, study approval

Copyright $($ C) 2018 Yeungnam University College of Medicine

This is an Open Access article distributed under the terms of the Creative Commons Attribution Non-Commercial License (http://creativecommons.org/licenses/by-nc/4.0/) which permits unrestricted non-commercial use, distribution, and reproduction in any medium, provided the original work is properly cited. 
from an Institutional Review Board (IRB) is essential based on the Korean Bioethics and Safety Act. However, in most cases, paraffin blocks are collected during the treatment process and consent obtained from the patients is for surgery and not for research. Consequently, decisions made by IRBs based on the Korean Bioethics and Safety Act are often not uniform. More specifically, different IRBs make different decisions based on the consent forms, and some studies are unable to proceed due to lack of approval. Therefore, we aim to suggest review points for studies using paraffin blocks based on the Korean Bioethics and Safety Act, in order to assist IRBs with a consistent and uniform review process and promote novel studies in the field.

\section{Studies utilizing paraffin blocks and legal requirements for IRB approval}

The definition of 'human biologic material' based on the Korean Bioethics and Safety Act revised in 2015 is 'all tissues, cells, bloods, or body fluids, as well as serum, plasma, chromosomes, DNA, ribonucleic acid, or proteins extracted from them (chapter 1, article 2)'. Paraffin blocks are made of cells and tissues extracted during surgery or biopsy and therefore are human derivatives. Consequently, the patient's consent as well as IRB approval is essential for research purposes.

\section{Studies utilizing paraffin blocks and issues regarding patient consent}

The Korean Bioethics and Safety Act article 16 (consent from human subjects for research) section 1 indicates that "investigators utilizing human samples must obtain written consent from the human subjects prior to the study". However, written consent may be waived in certain cases. Article 16 paragraph 3 indicates that "despite the content in section 1 , obtaining written consent from patients may be waived if the following conditions are met and IRB approval is obtained": 1) cases where obtaining written consent from the patient is practically impossible or will have severe effect on validity of the study; 2) cases where no evidence is provided to assume withdrawal of consent and rare or not greater than minimal risk would be put upon the patient even if s/he withdrew consent.

Human derivatives in paraffin blocks are biological speci- mens extracted or resected for diagnostic and therapeutic purposes, but not for research purposes. In other words, these are human specimens extracted with the patient's consent and can be discarded based on the Waste Management Act article 2 no. 5 (human biomaterial or extractions from medical institutions). Therefore, consent provided for surgery should also be sufficient for extraction and legal management of the extracted materials after the surgery. In addition, 'agreement for extraction' should be considered as 'the patient has given up on the extracted tissue'. Whether additional consent from the patient is required when using their tissue - which they have either given up or given consent to extract - for additional research purposes is questionable. Consent forms for utilizing human biomaterial required by the Korean Bioethics and Safety Act should initially reflect the use of extracted material for research purposes, separate from the cases where tissues are extracted for diagnosis and treatment initially and the remaining tissue is used for research purposes. The principles discussed and utilized by the American Society for Clinical Pathology also suggest that "the patient has given up on the extracted tissue", and the remaining specimen or paraffin blocks after diagnosis can freely be used for research purposes without an additional consent process [1].

However, other people argue that the consent provided by the patient for surgery is for discarding their extracted tissue after the surgery and not for utilizing the tissue for research purposes. Therefore, they suggest that approval for further research should be obtained initially and an additional consent is required if no appropriate consent was obtained initially. Some of the IRBs that support this idea conclude that "paraffin blocks archived in the pathology department were not approved for research and therefore cannot be used for research purposes [2]".

\section{Studies utilizing paraffin blocks and conditions for waived consent}

IRB should examine the study proposal and decide whether the study fulfills conditions for waived consent outlined in the Korean Bioethics and Safety Act article 16 paragraph 3 , instead of assuming that consent is essential for studies utilizing human biomaterial. Let us apply the condition 1) "cases where obtaining written consent from the patient is practically impossible or will have severe effect on validity 
of the study". In most studies using paraffin blocks, it is practically impossible to obtain written consent from the participant (patient who provided the specimen). This is because majority of studies using paraffin blocks utilize blocks that have been stored for several years and therefore it is difficult to be in touch with these patients. More specifically for paraffin blocks from malignant tumor tissues, the patient may have deceased. It is questionable whether contacting the patients by telephone or email, after searching for their personal information from medical records, just to obtain additional consent is ensuring the autonomy of these specimen providers - which is a fundamental bioethical principle. In fact, this may infringe on the patient's personal information and reveal sensitive information. Therefore, forcing the process of obtaining consent may be invading the patient's privacy. Moreover, majority of studies try to utilize as many tissue samples as possible to test statistical significance, and delay in studies due to the process of obtaining consent is inevitable. However, if an IRB decides that the study requires additional consent from the patients, large number of paraffin blocks being used in the study cannot be a reason for waived consent.

Moreover, the investigator should be able to explain that the study is fulfilling the conditions outlined in article 16 paragraph 3. 2) Cases where minimal physical or mental risks are put upon the patient even if additional consent not obtained. Since the study utilizes tissues extracted from surgery, there is no additional physical pain inflicted on the patients. In addition, there is no mental or social risk if there is no personal contact between the investigator and the patient. Therefore, an IRB can allow waived consent for the studies that do not utilize personal information, by utilizing anonymous data. Nonetheless, if the study requires special information or follow-up and consequently requires personal contact with the patient, an additional consent will be essential.

Medical Appliances Act allows for utilization of specimens for clinical trials without additional consent, if data anonymity is ensured. Article 24 paragraph 1 indicates that the following specimens can be used without consent form with anonymized data: "12. following specimens ("remaining specimen" from here on) being used in clinical trials without obtained consent from paragraph 1 no. 4 should be used with anonymized personal data for the specimen providers: A. specimen from remaining human derivatives originally extracted for diagnostic or therapeutic purposes; B. specimens from remaining human derivatives originally extracted for specific research purposes, being used for secondary purpose (with comprehensive consent)".

Newly published "guidelines for understanding the conditions for waived consent when assessing study protocols utilizing remaining specimens to be discarded in the hospital" by the department of bioethical policy in August 2017 has remedied these shortcomings [3]. This guideline also suggests that specimen can be used without additional consent, given that the data is anonymized.

\section{Issues with managing and storing paraffin blocks}

When evaluating the study protocols utilizing paraffin blocks, not only bioethical aspects including consent forms, but also the fact that paraffin blocks are stored as a type of medical record, should be considered. Current "Medical Legislation Enforcement Regulations" article 15 (maintenance of medical records) indicates that paraffin blocks should be a type of medical record like radiologic imaging, and minimum of 5 years storage is mandatory. Although paraffin blocks or pathological slides are not included in the regulation, they should also be managed and stored in the same way. The Korean Society of Pathology suggests that these materials should be stored for more than 5 years along with other medical records [4].

Forced storage of these tissue blocks not only preserves continuous diagnosis and treatment history of the patient but also allows them to act as a legal evidence. Furthermore, storing these paraffin blocks and slides allows additional consultation with experts from different institutes and novel diagnostic methods can be applied on these past samples. Therefore, the blocks to be used for research purposes should be assessed, regardless of whether it violates the patient's right to receive better diagnosis and treatment. If there are several blocks and only one is being used for the study, there should not be an issue. However, the investigator should not use the specimen if there is one remaining block for the patient or if there will be no remaining block after using the sections for the study [5]. In the study proposal, the investigators must agree that they will not use the block if 'there is one last remaining block for the patient' or they will obtain non-stainedglass slides if 'there is one last remaining block for the patient'. 
In addition, considering that enforced storage period for medical records is 5 years, one can claim that paraffin blocks older than 5 years may be used without an additional consent form.

\section{Studies using paraffin blocks and conditions for exempted IRB review}

Studies utilizing paraffin blocks, based on the content of the study, can be exempted for IRB review according to the Korean Bioethics and Safety Act article 36 paragraph 2. According to paragraph 2 no. 1, following cases can have IRB review exempted if "the investigator does not collect or record personal information": "A. studies utilizing human biomaterial and relevant genetic information collected and stored by the human biobank, where personal information cannot be assessed without going through the human biobank that provided the samples; B. studies that assess quality control of laboratories or quality assurance of data using remaining human biomaterial after diagnostic and therapeutic uses; C. studies that use separated/manufactured study material (i.e. pathogen, cell culture) from human biomaterial; and D. studies where the investigator does not have access to personal information of the specimen donor and no genetic relationship is found between study outcome and genetic information of the donor - except for studies using embryonic stem cells". In summary, paraffin block-utilizing studies where 'the investigator has no information on personal identification and the study is not investigating a hereditary trait' are exempted from review. However, the exemption of IRB review is not equivalent to waived consent obtaining and therefore these should be evaluated separately. There are claims suggesting that paraffin blocks should be considered as 'B. remaining human biomaterial after diagnoses' and can only be used for 'quality control', and therefore cannot be used for research purposes. This leads us to believe that the Korean Bioethics and Safety Act was focusing on the human biomaterial collected for research purpose, lacking the awareness of diverse human biomaterial produced for diagnostic and therapeutic purposes.

\section{CONCLUSION}

In this study, we have emphasized the value of paraffin blocks in medical research and summarized the possibility of waived written consent for paraffin block-utilizing studies under the Korean Bioethics and Safety Act. Current Act article 16 paragraph 3 suggests the possibility of waived written consent for studies utilizing paraffin blocks, and article 36 paragraph 2 demonstrates potential exemption of IRB review. Therefore, IRB should ask the investigator to explain that it is practically impossible to obtain written consent for the proposed study and there would be no potential threats or risks on the specimen provider even if the study proceeds without consent. Thereafter, validity of this explanation should be examined by the IRB. Lastly, considering that paraffin blocks are a type of medical record, the study investigator must ensure that the paraffin block is not damaged so that potential demands in the future by the specimen provider can be addressed.

\section{CONFLICT OF INTEREST}

No potential conflict of interest relevant to this article were reported.

\section{ORCID}

Yong-Jin Kim, https://orcid.org/0000-0002-9867-0752

\section{REFERENCES}

1. Dry S. Who owns diagnostic tissue blocks? Lab Med 2009;40: 69-73.

2. Information Portal of Institutional Review Boards designated by the Ministry of Health and Welfare. 100 questions and 100 answers. 2013 to 2014 collection of open writings 30 . Sejong: Ministry of Health and Welfare; 2015.

3. Department of Bioethics Policy, Ministry of Health and Welfare. Guidelines for understanding the conditions for waived consent when assessing study protocols utilizing remaining specimens to be discarded in the hospital [Internet]. Sejong: Ministry of Health and Welfare; 2017 [cited 2018 April 30]. https://bci.gilhospital.com/BlueAD/board.php?bbs_id=gc_notice\&bbs_no $=184 \&$ mode $=$ view\&type $=6$

4. Korean Society of Pathologists/Korean Society for Cytopatholgy. Pathology red book for quality assurance 2018 [Internet]. Seoul: Korean Society of Pathologists/Korean Society for Cytopatholgy; 2018. p. 25-6 [cited 2018 April 30]. http://www.pathology.or.kr/html/?pmode $=$ boardview\&MMC_pid=251\&seq $=18362$

5. Kim YJ, Park JS, Ko K, Jeong CR. Let archived paraffin blocks be utilized for research with waiver of informed consent. J Pathol Transl Med 2018;52:141-7. 